\title{
High-frequency viscosity and generalized Stokes-Einstein relations in dense suspensions of porous particles
}

\author{
Gustavo C. Abade*, ${ }^{1}$ Bogdan Cichocki, ${ }^{1}$ Maria L. \\ Ekiel-Jeżewska, ${ }^{2, \dagger}$ Gerhard Nägele, ${ }^{3}$ and Eligiusz Wajnryb ${ }^{2}$ \\ ${ }^{1}$ Institute of Theoretical Physics, University of Warsaw, Hoża 69, 00-681 Warsaw, Poland \\ ${ }^{2}$ Institute of Fundamental Technological Research, \\ Polish Academy of Sciences, Pawińskiego 5B, 02-106 Warsaw, Poland \\ ${ }^{3}$ Institut für Festkörperforschung, Forschungszentrum Jülich, D-52425 Jülich, Germany
}

(Dated: July 15, 2010)

\begin{abstract}
We study the high-frequency limiting shear viscosity, $\eta_{\infty}$, of colloidal suspensions of uncharged porous particles. An individual particle is modeled as a uniformly porous sphere with the internal solvent flow described by the Debye-Bueche-Brinkman equation. A precise hydrodynamic multipole method with a full account of many-particle hydrodynamic interactions encoded in the HYDROMUlTIPOLE program extended to porous particles, is used to calculate $\eta_{\infty}$ as function of porosity and concentration. The second-order virial expansion for $\eta_{\infty}$ is derived, and its range of applicability assessed. The simulation results are used to test the validity of generalized StokesEinstein relations between $\eta_{\infty}$ and various short-time diffusion coefficients, and to quantify the accuracy of a simplifying cell model calculation of $\eta_{\infty}$. An easy-to-use generalized Saitô formula for $\eta_{\infty}$ is presented which provides a good description of its porosity and concentration dependence.
\end{abstract}

PACS numbers: 82.70.Dd, 66.10.cg, 67.10.Jn

\footnotetext{
* present address: Departamento de Engenharia Mecânica, Faculdade de Tecnologia, Universidade de Brasília, Campus Universitário Darcy Ribeiro, 70910-900 Brasília-DF, Brazil
} 


\section{Introduction}

Suspensions of solvent-permeable colloidal particles are frequently encountered in colloid science, and are therefore the subject of ongoing research. Experimentally studied examples include thermosensitive microgel particles [1], soft giant micelles [2], and spherical core-shell particles consisting of a dry, solid core and an outer porous layer formed by a grated polymer brush [3]. The calculation of rheological and diffusion transport properties for porous particle systems is difficult since one has to deal with many-particle hydrodynamic interactions (HIs) mediated by the solvent flow outside and inside the particles. This is the reason why most theoretical work on dense suspensions was restricted so far to non-porous particles.

In this letter, we report on a detailed simulation study of the low-shear, high-frequency limiting shear viscosity, $\eta_{\infty}$, of a system of uniformly porous, non-overlapping spheres, as a function of particle volume fraction $\phi$ and porosity $k$. Our study covers the complete fluidphase concentration regime, and the whole range of particle porosities. The virial expansion of $\eta_{\infty}$ up to quadratic order in $\phi$ is derived for various porosities, and its range of applicability is assessed. We show that the porosity and concentration dependence of $\eta_{\infty}$ is well described by an easy-to-use generalized Saitô formula [4], and we point to the limited accuracy of a recent analytic cell model calculation [5]. Furthermore, we explore the validity of generalized Stokes-Einstein (GSE) relations between $\eta_{\infty}$ and various short-time diffusion coefficients, including the translational self-diffusion coefficient $D_{s}$, the sedimentation coefficient, $K$, and the cage diffusion coefficient $D\left(q_{m}\right)$. These short-time diffusion properties have been additionally explored by us in a recent simulation study $[6,7]$. It is shown in the following that the considered GSE relations are of limited use only.

\section{Model and simulation method}

We describe the creeping solvent flow outside and inside a sphere of radius $a$ and uniform porosity $k$ by the Stokes and Debye-Bueche-Brinkman (DBB) [8,9] equations, respectively,

$$
\begin{aligned}
\eta_{0} \nabla^{2} \boldsymbol{v}(\boldsymbol{r})-\eta_{0} \kappa^{2}\left[\boldsymbol{v}(\boldsymbol{r})-\boldsymbol{u}_{i}(\boldsymbol{r})\right] \chi(\boldsymbol{r})-\nabla p(\boldsymbol{r}) & =0 \\
\boldsymbol{\nabla} \cdot \boldsymbol{v}(\boldsymbol{r}) & =0 .
\end{aligned}
$$

Here, $\mathbf{v}$ and $\mathbf{p}$ are the fluid velocity and the pressure fields, and $\kappa^{-1}=\sqrt{k}$ is the hydrodynamic penetration depth. The characteristic function, $\chi(\mathbf{r})$, is non-zero and equal to one 
for $\mathbf{r}$ inside an arbitrary sphere $i$ only. The skeleton of the sphere moves rigidly according to $\mathbf{u}_{i}(\mathbf{r})=\mathbf{U}_{i}+\boldsymbol{\omega}_{i} \times\left(\mathbf{r}-\mathbf{R}_{i}\right)$, with the translational and rotational velocities $\mathbf{U}_{i}$ and $\boldsymbol{\omega}_{i}$, respectively. To determine $\eta_{\infty}$ and other transport properties, requires to solve the flow equations inside and outside the spheres, with the boundary conditions that $\mathbf{v}$ and the solvent stress change continuously across the sphere surfaces. The DBB equation for the intra-particle flow applies when the mean pore size, $\delta \propto \kappa^{-1}$, of the skeleton is sufficiently smaller than the particle radius $a$, i.e., when the dimensionless inverse penetration depth, $x=\kappa a$, is sufficiently large (i.e., $x \geq 5$ ). In the zero-penetration limit $x \rightarrow \infty$, hard spheres with stick surface boundary conditions are described. The model of homogeneously porous spheres is fully characterized by $x$ and the volume fraction $\phi=4 \pi a^{3} n / 3$, dependent on the particle radius $a$ and the particle-number concentration $n$.

We have calculated $\eta_{\infty}$ to high precision using a hydrodynamic multipole method corrected for lubrication [12-15], encoded in the HYDROMULTIPOLE program package. The hydrodynamic structure of the particles enters into the HYDROMULTIPOLE code through a single-particle friction operator only, whose form is known for a variety of particle models with different internal hydrodynamic structures $[12,16]$. The details of our simulation method are given elsewhere [6]. The transport coefficients discussed in this letter have been obtained from equilibrium configuration averages over typically $N=256$ particles in a periodically replicated cubic simulation box, with the hydrodynamic multipole order $L$ truncated usually at $L=3$. To gain high-precision data, an extrapolation procedure to $L \rightarrow \infty$ has been applied. A finite system size extrapolation formula to the thermodynamic limit $N \rightarrow \infty$ by Ladd [17] was used to calculate the diffusion properties in [7]. As pointed out already by Ladd [17], the simulated values for $\eta_{\infty}$ are not critically dependent on finite size scaling. The remaining error in $\eta_{\infty}$ and in the diffusion coefficients has been reduced in this way to less than $1 \%$.

The high-frequency viscosity linearly relates the average deviatoric suspension stress in an isotropic system to the applied high-frequency oscillatory rate of strain. In the linear response regime of low shear considered here, $\eta_{\infty}$ is determined by the equilibrium particle distribution of the unsheared system. The statistical mechanical expression for $\eta_{\infty}$ is [18]

$$
\eta_{\infty}=\eta_{0}+\frac{1}{10 V}\left\langle\sum_{i, j=1}^{N} \boldsymbol{\mu}_{i j, \alpha \beta \beta \alpha}^{d d}\right\rangle^{p b c}
$$

where Cartesian components of a $3 \times 3 \times 3 \times 3$ dipole-dipole mobility matrix $\boldsymbol{\mu}_{i j}^{d d}$ linearly 
relate the symmetric hydrodynamic force dipole moments acting on the surface of sphere $i$ to the rate of strain applied to sphere $j$, and the summation with respect to $\alpha$ and $\beta$ is included in Eq. (2). The generalized self- and distinct mobility matrices, $\boldsymbol{\mu}_{i j}^{d d}$, are evaluated from the multipole expansion method (see, e.g., $[4,18,19])$. The average, $\langle\ldots\rangle^{p b c}$, is taken over random equilibrium configurations of $N$ spheres in the basic simulation cell of volume $V$, with periodic boundary conditions.

We point out here that the high-frequency limiting viscosity $\eta_{\infty}$ is influenced by excluded volume interactions only through the equilibrium averaging. The extensive analysis of the influence of different types of interactions, i.e. direct and hydrodynamic ones, is discussed elsewhere [20].

The high-frequency viscosity should be distinguished from the static or zero-frequency viscosity, $\eta=\eta_{\infty}+\Delta \eta$, which has an additional contribution, $\Delta \eta>0$, arising from the time-integrated relaxation of the shear-distorted particles microstructure. The so-called relaxation term $\Delta \eta$ depends both on direct and hydrodynamic interactions. It is similar to the collision viscosity contribution in an atomic fluid. For the latter, however, there is no solvent and hence no hydrodynamic interactions.

\section{Two-body hydrodynamics}

At a small volume fraction, the suspension viscosity $\eta_{\infty}$ can be expanded in a virial series as $[4]$

$$
\eta_{\infty}(x, \phi)=\eta_{0}\left(1+[\eta] \phi+k_{H}[\eta]^{2} \phi^{2}+\mathcal{O}\left(\phi^{3}\right)\right)
$$

where $\eta_{0}$ is the solvent viscosity. Moreover,

$$
[\eta]=\frac{5}{2} \Omega_{v}(x)=\frac{5}{2}\left(\frac{G(x)}{1+10 G(x) / x^{2}}\right)
$$

with the function $G(x)=1+3 / x^{2}-3 \operatorname{coth}(x) / x$, is the intrinsic viscosity of porous spheres first derived in [9]. Eq. (4) generalizes Einstein's result for the intrinsic viscosity of nonporous spheres to porous ones. The original Einstein's result, $[\eta]=5 / 2$, is reproduced here in the limit $x \rightarrow \infty$. The function $\Omega_{v}(x)=\left(a_{\mathrm{eff}} / a\right)^{3}$ is the ratio of the intrinsic viscosities of non-porous and porous spheres. It is sometimes used to define a porosity-dependent, effective hard-sphere diameter, $a_{\mathrm{eff}}(x)<a$, with the associated effective volume fraction 
$\phi_{\text {eff }}(x)=\phi \Omega_{v}(x)=(2 / 5)[\eta] \phi[10,11]$. The Huggins coefficient, $k_{H}$, is determined by twobody hydrodynamic contributions using the method described in Ref. [20]. For non-porous hard spheres, Batchelor and Green [21] obtained $k_{H}[\eta]^{2}=5.2$, an approximate value that was improved in later work by Cichocki and Felderhof [22] to the high-precision result of 5.00. The Huggins coefficient for porous spheres has not been evaluated so far. Therefore, Table I includes our calculated values for the Huggins coefficient as a function of $x$.

TABLE I: The Huggins coefficient $k_{H}$ and the second virial coefficient $k_{H}[\eta]^{2}$ as functions of $x$.

\begin{tabular}{cccccccc}
\hline \hline $\mathrm{x}$ & 5 & 10 & 20 & 30 & 50 & 100 & $\infty$ \\
\hline$k_{H}$ & 0.53205 & 0.62703 & 0.70191 & 0.73231 & 0.75872 & 0.77957 & 0.80037 \\
$k_{H}[\eta]^{2}$ & 0.61607 & 1.8139 & 3.0918 & 3.6610 & 4.1693 & 4.5783 & 5.0023 \\
\hline \hline
\end{tabular}

\section{Simulation results}

Fig. 1 includes our simulation results for the concentration dependence of $\eta_{\infty} / \eta_{0}$, for the indicated values of $x$. Porosity allows the fluid to flow through the particles. The extra stress and viscous dissipation in the fluid caused by the particles is thus smaller at larger porosity. As a consequence, $\eta_{\infty}$ decreases with increasing porosity (decreasing $x$ ). The decrease of the viscosity with increasing porosity is quite substantial at larger $\phi$. The influence of porosity on $\eta_{\infty}$ should be easily detectable experimentally. For example, in a suspension of particles at $\phi=0.35$ with a typical mean porosity of $x \approx 30[3], \eta_{\infty}$ is about $30 \%$ lower than at zero porosity.

Our results for $\eta_{\infty} / \eta_{0}$ are in agreement with those of Mo and Sangani [23] in the more restricted range of $x \leq 20$ values considered in their study. For porous particles with $x \leq 20$, the largest differences between their values and our findings, detected at higher volume fractions, are of the order of $1 \%$. Slightly larger differences are expected for larger values of $x$. In the limiting case of $x=\infty$, i.e. for hard non-porous spheres, and $\phi=0.35$ and 0.45 , the results given in Ref. [23] underestimate the values of $\eta_{\infty} / \eta_{0}$ by $6 \%$ and $4 \%$, respectively. This inaccuracy is caused by the small number, $N=16$, of particles in the basic simulation cell; $N=16$ is too low to reproduce the correct equilibrium correlations. However, our viscosity data for the special case of non-porous spheres agree well with earlier 
force multipole simulation results by Ladd [17], who investigated larger numbers of particles in the periodic cell, $N \leq 108$, and with the accelerated Stokesian dynamics simulation data by Sierou and Brady [24] and Banchio and Nägele [25].
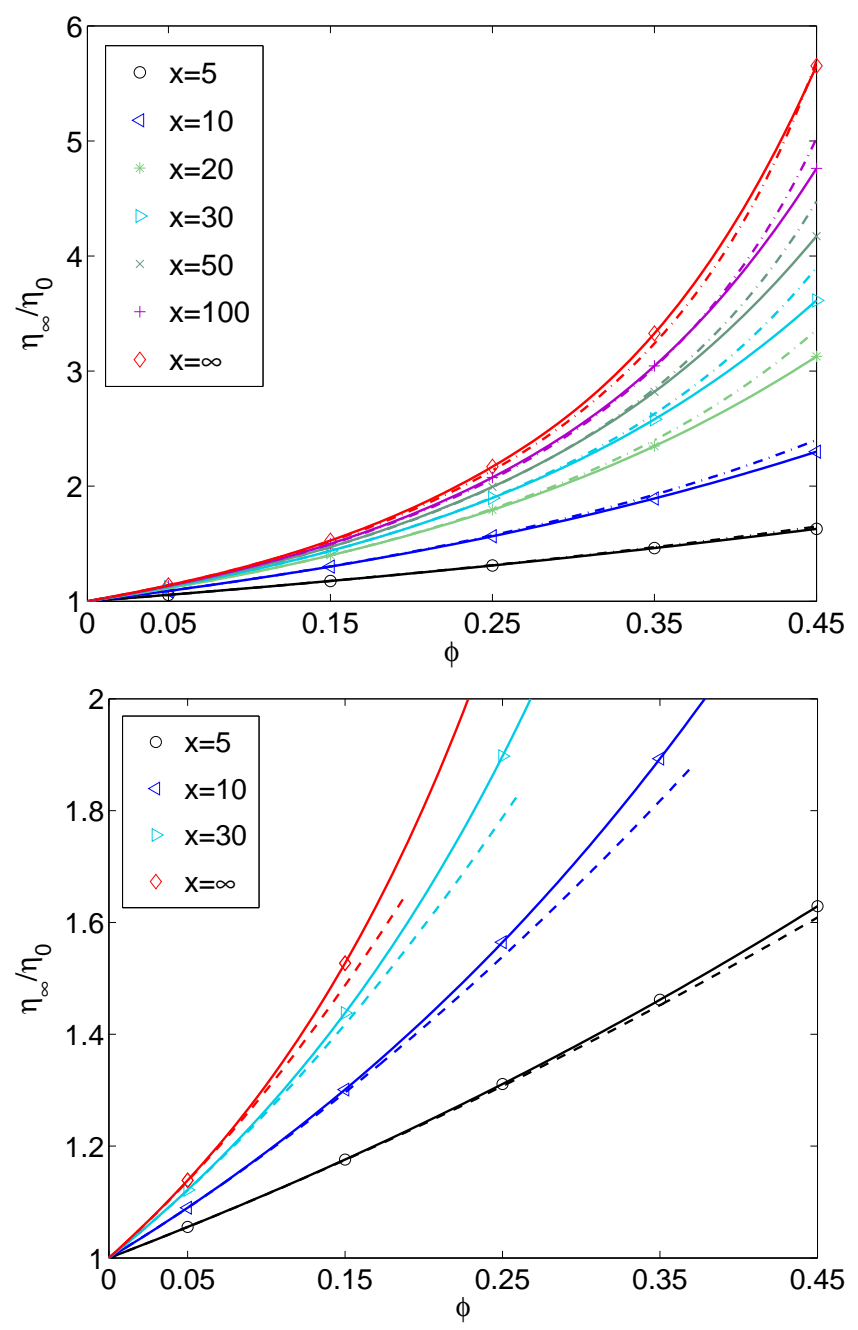

FIG. 1: High-frequency viscosity, $\eta_{\infty} / \eta_{0}$, for different porosities $(x=\infty$ corresponds to nonporous hard spheres). Simulation results (symbols) are connected by solid lines (splines) to guide the eye. Top: Comparison with the generalized Saitô expression, Eqs. (5)-(6) (dashed-dotted lines). Bottom: Comparison with the second-order viral expansion in Eq. (3) (dashed lines).

Using our results for $k_{H}(x)$, we can obtain an approximate analytic expression for $\eta_{\infty}$, valid at larger $\phi$, from the generalized Saitô formula [4]

$$
\eta_{\infty} / \eta_{0}=1+[\eta] \phi \frac{1+S}{1-\frac{2}{5}[\eta] \phi(1+S)}
$$

which expresses $\eta_{\infty}$ in terms of the intrinsic viscosity $[\eta]$ and a function $S(x, \phi)$. A micro- 
scopic expression for $S$ has been derived in [4]. Since $S$ is more amenable to a low-density approximation than $\eta_{\infty}$, we equate the first-order concentration expansions of Eqs. (5) and (3). This leads to a useful approximation for $S$ by

$$
S=\left(k_{H}-\frac{2}{5}\right)[\eta] \phi
$$

in terms of the one-body and two-body properties $[\eta]$ and $k_{H}$, respectively, which depend only on $x$.

Fig. 1 includes additionally the viscosity prediction by our generalized Saitô formula in Eqs. (5-6) for the full range $\phi \leq 0.45$, with $[\eta]$ according to Eq. (4) and the values for $k_{H}(x)$ taken from table I. The overall agreement with the simulation data is good, in particular at very small and very large values of $x$; Eqs. (5) and (6) describe the high-frequency viscosity of porous spheres quite well. At intermediate values of $x$ and large $\phi$, the viscosity is somewhat overestimated. That the generalized Saitô formula is more useful than the direct 2nd-order virial expansion, for the same input $[\eta]$ and $k_{H}$, can be clearly noticed from Fig. 1 (bottom). The simulation data for $\phi>0.2$ are well-described by the 2 nd-order virial form only for a large porosity (i.e., small value of $x$ ). This can be explained by the weakening of the strength of the HIs with increasing porosity, which is most pronounced regarding the three-body and higher-order hydrodynamic contributions [26].

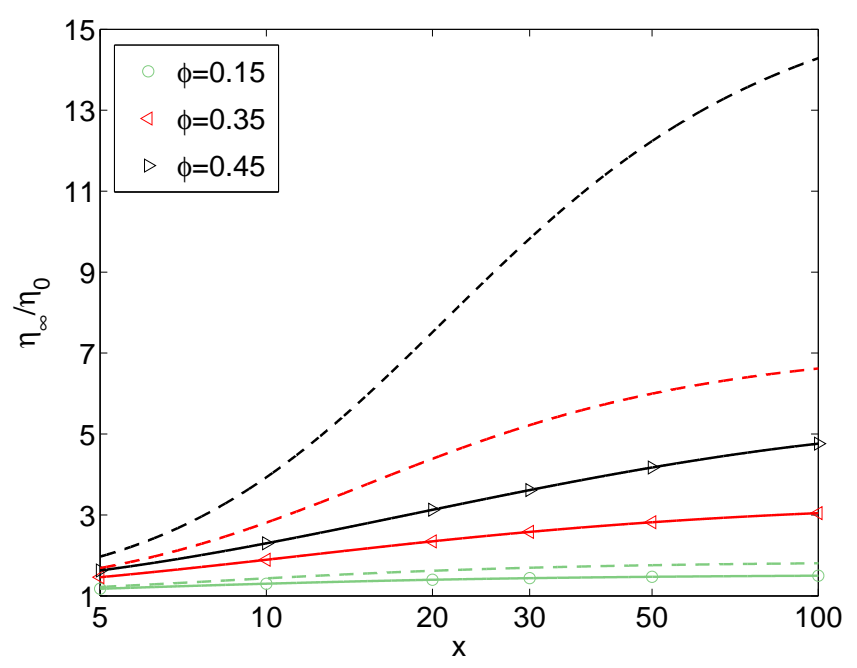

FIG. 2: High-frequency viscosity, $\eta_{\infty} / \eta_{0}$, as a function of the inverse hydrodynamic penetration depth $x$. Comparison of the simulation results (symbols connected by splines) with the spherical cell model prediction from [5] (dashed lines). Each color corresponds to a different volume fraction. 
We proceed by comparing our simulation data for $\eta_{\infty}$ with approximate spherical cell model calculation results. Quite recently, Ohshima derived an explicit analytic cell model expression for the high-frequency viscosity of uncharged porous spheres as a function of $\phi$ and $x$ (see his Eq. (51) in [5], with $x$ identified as $\lambda a$ ). Spherical cell model calculations are frequently used to this date to predict the primary electroviscous effect on the high-frequency viscosity in non-dilute suspensions of charged porous [27] and non-porous [28] spheres with weakly overlapping electric double layers. In the cell model, the complicated hydrodynamic and electrokinetic influence by the (microion-dressed) colloidal spheres surrounding a central one, is accounted for in a very crude way through the concentration dependence, $R=$ $a \phi^{-1 / 3}$, of the outer boundary radius $R$, and the specified outer boundary conditions. This strong simplification allows for an analytic solution for $\eta_{\infty}(x, \phi)$. The accuracy of Ohshima's expression for $\eta_{\infty}$ is examined in Fig. 2. It is exact to first order in $\phi$ only. At larger $\phi$ where inter-particle correlations come into play, Ohshima's expression strongly overestimates the high-frequency viscosity, and this overestimation is most pronounced at lower porosity. The comparison with our simulation data indicates that the fluid-like inter-particle correlations neglected in the cell model should play a decisive role also for charged colloidal spheres.

\section{Generalized Stokes-Einstein relations}

In recent related work $[6,7]$, we have calculated the short-time diffusion properties of suspensions of homogeneously porous spheres using the HYDROMULTIPOLE simulation method. All the diffusion coefficients studied in our earlier work are related to the short-time diffusion function,

$$
D(q)=D_{0} H(q) / S(q)
$$

measurable as a function of the scattering wavenumber $q$ in a dynamic scattering experiment [25]. The diffusion function is equal to the ratio of the hydrodynamic function, $H(q)$, and the equilibrium static structure factor, $S(q)$, multiplied by the diffusion coefficient, $D_{0}(x)$, of a single porous sphere [29]. The function $H(q)$ encodes the influence of HIs on short-time diffusion. In the limit of $q \rightarrow \infty, H(q)$ is equal to the normalized short-time self-diffusion coefficient, $D_{s} / D_{0}$. At $q \rightarrow 0, H(q)$ reduces to the sedimentation coefficient $K=U / U_{0}$, where $U$ is the mean sedimentation velocity of a homogeneous suspension of weakly sedimenting porous particles, with $U_{0}$ denoting the velocity at infinite dilution. The 
cage diffusion coefficient, $D\left(q_{m}\right)$, characterizes short-time diffusive motion on a length scale $\sim 2 \pi / q_{m}$, comparable to the nearest neighbor shell distance. At the wavenumber $q_{m}, S(q)$ attains its principal maximum. Our simulation results for $H(q)$, and the associated diffusion and sedimentation coefficients $D_{s}, D\left(q_{m}\right)$ and $K$ have been thoroughly discussed in $[6,7]$.
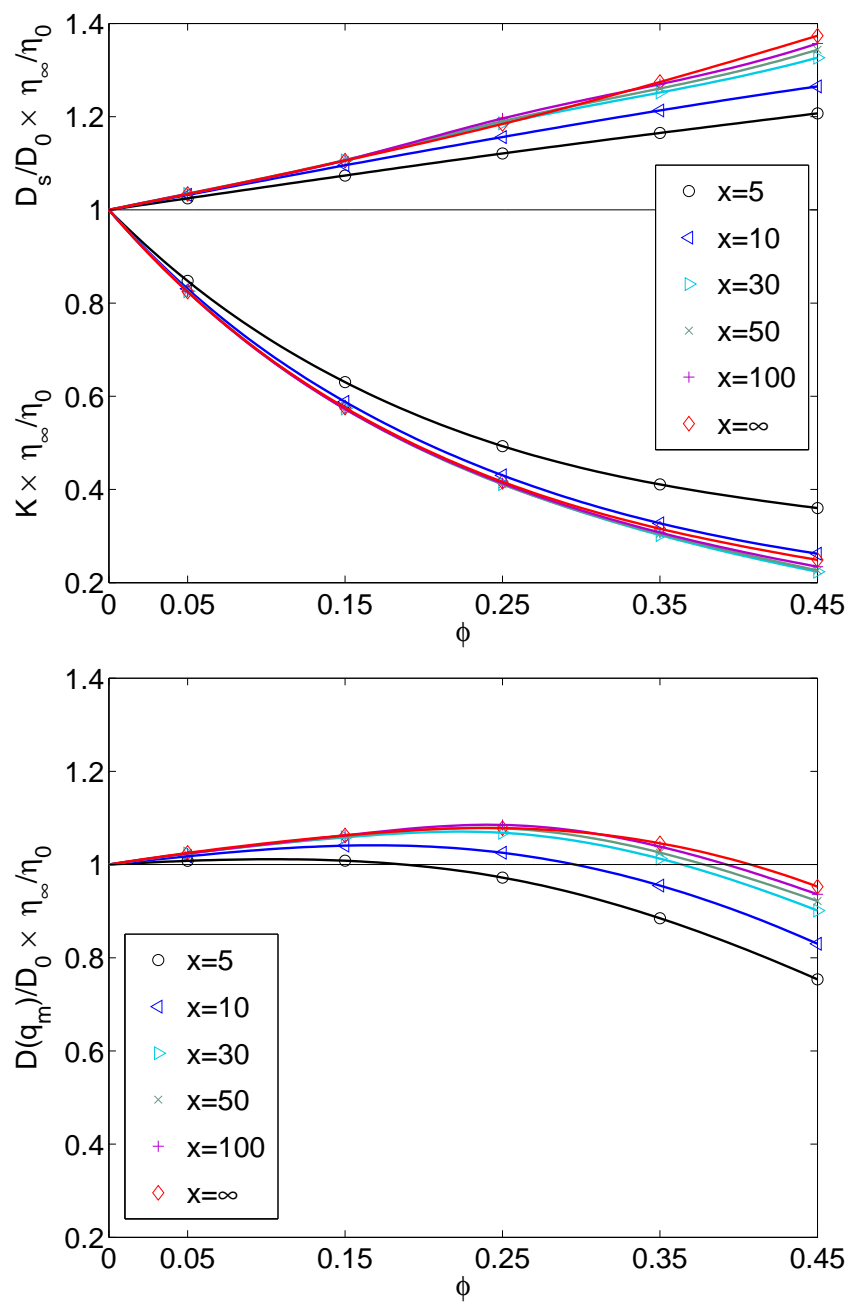

FIG. 3: Test of three generalized Stokes-Einstein (GSE) relations for the high-frequency viscosity $\eta_{\infty} / \eta_{0}$ multiplied by the sedimentation coefficient $K$, normalized self-diffusion coefficient $D_{s} / D_{0}$, and normalized cage diffusion coefficient $D\left(q_{m}\right) / D_{0}$, respectively. Top: For larger $\phi$, GSE scaling does not apply to $K$ and hardly so to $D_{s} / D_{0}$. Bottom: GSE scaling is approximately valid for $D\left(q_{m}\right) / D_{0}$, with an accuracy better than $10 \%$ up to $\phi \approx 0.3$.

We are here in the position to analyze, for the case of uniformly porous particles, the relation between $\eta_{\infty}$ and the diffusion and sedimentation coefficients $D_{s}, D\left(q_{m}\right)$ and $K$, 
respectively. Specifically, we test here the validity of the following three GSE relations

$$
\frac{D(x, \phi)}{D_{0}(x)} \cdot \frac{\eta_{\infty}(x, \phi)}{\eta_{0}} \approx 1
$$

with $D$ given by $D=D_{s}, D=D\left(q_{m}\right)$ or $D=D_{0} K$, respectively. These relations are exact at $\phi=0$ only. If a GSE relation were valid to decent accuracy at non-zero $\phi$, it would be quite useful from an experimental viewpoint, since the viscosity could be determined then more easily in a scattering experiment.

In Fig. 3, the validity of the GSE relations is examined for different values of $x$, with the simulation data for $D_{s}, D\left(q_{m}\right)$ and $K$ taken from our previous study [6]. A valid GSE relation is reflected by a horizontal curve of unit distance above the concentration axis. For larger $\phi$ and all considered porosities, the GSE scaling in Eq. (8) does not apply for $K$, and it performs only a little better for $D_{s} / D_{0}$. Notice here that the product $D_{s} \eta_{\infty}$ is well approximated, for all $\phi$, by its first-order in $\phi$ expansion, reflected by the straight lines in Fig. 3 (top part). Interestingly enough, $K \eta_{\infty}$ is only moderately porosity dependent for $x \geq 10$, and for $\phi$ no larger than 0.35 .

Contrary to $D_{s}$ and $K$, GSE scaling for the cage diffusion coefficient is approximately satisfied (see Fig. 3, bottom part), with an accuracy better than $10 \%$ up to $\phi \approx 0.3$. Thus, for systems of electrically neutral (porous) particles where the amount of colloidal material is too low or too expensive to perform a mechanical rheological experiment, one can infer $\eta_{\infty}$ approximately but straightforwardly from a standard scattering experiment measurement of $D\left(q_{m}\right)$ using a first cumulant analysis [34].

The violation of the GSE relations for $K$ and $D_{s}$, and its only moderate accuracy for $D\left(q_{m}\right)$, reflect the different physical mechanisms underlying sedimentation, self-diffusion and cage diffusion, for non-zero concentrations when HIs are active. In [25], a short-time GSE analysis similar to the present one was made for non-porous, charged colloidal spheres at low-salt conditions. For charged spheres, the GSE relation for $D\left(q_{m}\right)$ was found to be strongly violated. This illustrates the general observation that the (approximate) validity of a GSE relation depends strongly on the range and character of the particle interactions. 


\section{Summary and outlook}

In summary, using the precise simulation method encoded in the HYDROMULTIPOLE program, we have studied the porosity and concentration dependence of $\eta_{\infty}$, a quantity routinely measured, e.g., using a torsional resonator viscometer operated at a small applied stress amplitude [30-33]. The second-order virial expansion for $\eta_{\infty}$ was derived by taking two-body HIs into account. It was used to derive a generalized and easy-to-use Saitô expression which describes the viscosity simulation data quite well. Precise values of the Huggins coefficient $k_{H}(x)$, which is the only non-trivial input in the generalized Saitô expression, are given in table I for an extended, representative set of porosity values. The influence of porosity on $\eta_{\infty}$ is found to be significant at larger concentrations, so that it can be detected experimentally.

The accuracy of various short-time generalized Stokes-Einstein relations, and of a cell model expression for $\eta_{\infty}$ have been tested. It was shown that the cell model gives a rather poor prediction for the (high-frequency) viscosity of non-dilute systems. This is an important finding since the spherical cell model is frequently used to this day to study the primary electroviscous effect on the (high-frequency) viscosity in concentrated suspensions of porous and non-porous charged particles. Moreover, we have shown that using the GSE relation between $\eta_{\infty}$ and $D\left(q_{m}\right)$, one can infer approximately the $\eta_{\infty}$ of neutral, porous spheres from the measurement of $D\left(q_{m}\right)$. The latter can be conveniently measured in a short-time dynamic scattering experiment. This is useful when the sample is too small in volume to perform a direct rheological measurement.

Finally, we emphasize that short-time transport properties are interesting not only in their own right. Their knowledge is also indispensable for a better understanding of the suspension dynamics at long times [34].

G.N., M.L.E.J. and B.C. acknowledge support by the Deutsche Forschungsgemeinschaft (SFB-TR6, project B2). M.L.E.J., E.W. and G.C.A. were supported in part by the Polish Ministry of Science and Higher Education grant 45/N-COST/2007/0; M.L.E.J. and E.W. also by the COST P21 Action "Physics of droplets". The work of G.C.A. was supported by CAPES Foundation/Ministry of Education of Brazil. Numerical calculations were done at the Academic Computer Center in Gdańsk, Poland. 
$\dagger$ Electronic address: mekiel@ippt.gov.pl

[1] T. Eckert and W. Richtering, J. Chem. Phys. 129, 124902 (2008).

[2] R. Sigel et al., Phys. Rev. Lett. 83, 4666 (1999).

[3] M.H.G. Duits, P. A. Nommensen, D. van den Ende, and J. Mellema, Colloids Surf. A 183-185, 335 (2001).

[4] B. Cichocki, B.U. Felderhof and R. Schmitz, Physica A 154, 233 (1989).

[5] H. Ohshima, Colloids and Surfaces A: PhysicoChem. Eng. Aspects 347, 33 (2009).

[6] G.C. Abade, B. Cichocki, M.L. Ekiel-Jeżewska, G. Nägele and E. Wajnryb, J. Chem. Phys. 132, 014503 (2010).

[7] G.C. Abade, B. Cichocki, M.L. Ekiel-Jeżewska, G. Nägele and E. Wajnryb, Phys. Rev. E 81, 020401(R) (2010).

[8] H.C. Brinkman, Appl. Scient. Res. A1 27, 81 (1947).

[9] P. Debye and A.M. Bueche, J. Chem. Phys. 16, 573 (1948).

[10] W. van Saarloos, Physica 147A, 280 (1987).

[11] L. Gmachowski, Colloids and Surfaces A: Physicochem. Eng. Aspects, 215, 173 (2003).

[12] B. Cichocki, B. U. Felderhof and R. Schmitz, PhysicoChemical Hydrodynamics 10, 383 (1988).

[13] B. Cichocki, B.U. Felderhof, K. Hinsen, E. Wajnryb, and J. Bławzdziewicz, J. Chem. Phys. 100, 3780 (1994).

[14] B. Cichocki, M.L. Ekiel-Jezewska and E. Wajnryb, J. Chem. Phys. 111, 3265 (1999).

[15] B. Cichocki, R.B. Jones, R. Kutteh and E. Wajnryb, J. Chem. Phys. 112, 2548 (2000).

[16] B. Cichocki and B. U. Felderhof, J. Chem. Phys. 130, 164712 (2009).

[17] A.J.C. Ladd, J. Chem. Phys. 93, 3484 (1990).

[18] P. Szymczak and B. Cichocki, J. Stat. Mech.: Theory Exp., P0125 (2008).

[19] B. Cichocki, M.L. Ekiel-Jezewska and E. Wajnryb, J. Chem. Phys. 119, 606 (2003).

[20] G. C. Abade, B. Cichocki, M. L. Ekiel-Jeżewska, G. Nägele, and E. Wajnryb, J. Chem. Phys., submitted.

[21] G.K. Batchelor and J.T. Green, J. Fluid Mech. 56, 401 (1972).

[22] B. Cichocki and B.U. Felderhof, J. Chem. Phys. 89, 1049 (1988).

[23] G. Mo and A. S. Sangani, Phys. Fluids 6, 1637 (1994). 
[24] A. Sierou and J.F. Brady, J. Fluid Mech. 448, 115 (2001).

[25] A.J. Banchio and G. Nägele, J. Chem. Phys. 128, 104903 (2008).

[26] We note here that the simulation data for $\eta_{\infty}$ at $x=\infty$ are well reproduced, for all $\phi$ where the system behaves fluid-like, by a fitting formula due to Ladd [17], also obtained from the Saitô expression in Eq. (5) by setting $[\eta]=5 / 2$ and $S(\infty, \phi)=\phi+\phi^{2}-2.3 \phi^{3}$.

[27] V. Natraj and S.B. Chen, J. Colloid Interface Sci. 251, 200 (2002).

[28] E. Ruiz-Reina and F. Carrique, J. Phys. Chem. C 111, 141 (2007).

[29] G. Neale, N. Epstein and W. Nader, Chem. Eng. Sci. 28, 1865 (1973).

[30] J. Bergenholtz, F.M. Horn, W. Richtering, N. Willenbacher and N.J. Wagner, Phys. Rev. E 58, R4088 (1998).

[31] J. Bergenholtz, N. Willenbacher, N.J. Wagner, B. Morrison, D. van den Ende and J. Mellema, J. Colloid Interface Sci. 202, 430 (1998).

[32] T. Shikita and D.S. Pearson, J. Rheol. 38, 601 (1994).

[33] G. Fritz, B.J. Maranzano, N.J. Wagner and N. Willenbacher, J. Non-Newtonian Fluid Mech. 102, 149 (2002).

[34] P. Holmqvist and G. Nägele, Phys. Rev. Lett. 104, 058301 (2010). 